\title{
PROBABILISTIC DERIVATION OF A BILINEAR SUMMATION FORMULA FOR THE MEIXNER-POLLACZEK POLYNOMIALS
}

\author{
P. A. LEE \\ Department of Mathematics \\ University of Malaya \\ Kuala Lumpur 22-11 \\ Malaysia
}

(Received August 24, 1978)

ABSTRACT. Using the technique of canonical expansion in probability theory, a bilinear summation formula is derived for the special case of the MeixnerPollaczek polynomials $\left.{ }_{[} \lambda_{n}^{(k)}(x)\right\}$ which are defined by the generating function $\sum_{n=0}^{\infty} \lambda_{n}^{(k)}(x) z^{n} / n !=(1+z)^{\frac{1}{2}(x-k)} /(1-z)^{\frac{1}{2}(x+k)},|z|<1$.

These polynomials satisfy the orthogonality condition

$$
\int_{-\infty}^{\infty} p_{k}(x) \lambda_{m}^{(k)}(i x) \lambda_{n}^{(k)}(i x) d x=(-1)^{n}{ }_{n} !(k){ }_{n} \delta_{m, n}, \quad i=\sqrt{-1}
$$

with respect to the weight function

$$
\begin{aligned}
p_{1}(x)= & \operatorname{sech} \pi x \\
p_{k}(x)= & \int_{-\infty}^{\infty} \cdots \int_{-\infty}^{\infty} \operatorname{sech} \pi x_{1} \operatorname{sech} \pi x_{2} \ldots \\
& \operatorname{sech} \pi\left(x-x_{1}-\ldots-x_{k-1}\right) d x_{1} d x_{2} \ldots d_{k-1}, k=2,3, \ldots
\end{aligned}
$$


KEY WOROS AND PHRASES. Meixner-Pollaczek polynomials, orthogonal polynomials, bilinear summation formula, bivariate distribution, canonical expansion, Runge identity, G-functions.

1980 MATHEMATICS SLBJECT CLASSIFICATION CODES. Primary 42A60, Secondary $33 A 70$ 62H2O.

\section{INTRODUCTION}

Let $U$ be a Cauchy random variable with the probability density function (p.d.f.)

$$
f(u)=\frac{1}{\pi} \frac{1}{1+u^{2}},-\infty<u<\infty .
$$

Consider the transformation $U=\sinh \pi \mathrm{V}$. The p.d.f. of $\mathrm{V}$ is

$$
\mathrm{p}(\mathrm{v})=\operatorname{sech} \pi \mathrm{v},-\infty<\mathrm{v}<\infty .
$$

This is the hyperbolic secant distribution considered by Baten [2], and is a special case of the generalized hyperbolic secant distribution treated by Harkness and Harkness [10].

Let $x_{1}$ and $x_{2}$ be two random variables having additive random elements in common [6], i.e.

$$
\begin{aligned}
& \mathrm{x}_{1}=\mathrm{v}_{1}+\mathrm{v}_{2} \\
& \mathrm{x}_{2}=\mathrm{v}_{2}+\mathrm{v}_{3}
\end{aligned}
$$

where $v_{i}(i=1,2,3)$ are mutually independent random variables each having the p.d.f. given in (1). The joint p.d.f. $p\left(x_{1}, x_{2}\right)$ of $x_{1}$ and $x_{2}$ is easily shown to be

$$
\begin{aligned}
\mathrm{p}\left(\mathrm{x}_{1}, \mathrm{x}_{2}\right) & =\int_{-\infty}^{\infty} \operatorname{sech} \pi \mathrm{z} \operatorname{sech} \pi\left(\mathrm{x}_{1}-\mathrm{z}\right) \operatorname{sech} \pi\left(\mathrm{x}_{2}-\mathrm{x}_{1}+\mathrm{z}\right) \mathrm{dz} \\
& =\frac{1}{2} \operatorname{sech} \frac{\pi \mathrm{x}_{1}}{2} \operatorname{sech} \frac{\pi \mathrm{x}_{2}}{2} \operatorname{sech} \frac{\pi\left(\mathrm{x}_{2}-\mathrm{x}_{1}\right)}{2}, \quad \begin{array}{l}
-\infty<\mathrm{x}_{1}<\infty, \\
-\infty<\mathrm{x}_{2}<\infty .
\end{array}
\end{aligned}
$$

The marginal p.d.f.'s for $x_{1}$ and $x_{2}$ are respectively 


$$
\begin{aligned}
& g\left(x_{1}\right)=\int_{-\infty}^{\infty} p\left(x_{1}, x_{2}\right) d x_{2}=2 x_{1} \operatorname{cosech} \pi x_{1}, \\
& h\left(x_{2}\right)=\int_{-\infty}^{\infty} p\left(x_{1}, x_{2}\right) d x_{1}=2 x_{2} \operatorname{cosech} \pi x_{2} .
\end{aligned}
$$

The orthogonal polynomials with the above marginals as weight function are related to the Euler numbers and have been discussed by Carlitz in [4]. Specifica11y, for the weight function

$$
\begin{aligned}
& \mathrm{p}_{\mathrm{k}}(\mathrm{x})=\int_{-\infty}^{\infty} \ldots \int_{-\infty}^{\infty} \operatorname{sech} \pi \mathrm{x}_{1} \operatorname{sech} \pi \mathrm{x}_{2} \ldots \operatorname{sech} \pi\left(\mathrm{x}-\mathrm{x}_{1}-\mathrm{x}_{2}\right. \\
& \left.\ldots . \mathrm{x}_{\mathrm{k}-1}\right) \mathrm{dx_{1 }} d \mathrm{dx}_{2} \ldots \mathrm{dx}_{\mathrm{k}-1}, \mathrm{k}=2,3, \ldots . \\
& \mathrm{p}_{1}(\mathrm{x})=\operatorname{sech} \pi \mathrm{x},
\end{aligned}
$$

the polynomials $\left\{\lambda_{n}^{(k)}(x)\right\}$ with generating function

$$
\sum_{n=0}^{\infty} \lambda_{n}^{(k)}(x) z^{n} / n !=(1+z)^{\frac{1}{2}(x-k)} /(1-z)^{\frac{1}{2}(x+k)},|z|<1
$$

are the orthogonal polynomials in the interval $(-\infty, \infty)$ and satisfy the orthogonality condition

$$
\int_{-\infty}^{\infty} p_{k}(x) \lambda_{m}^{(k)}(i x) \lambda_{n}^{(k)}(i x) d x=(-1)^{n} n !(k){ }_{n} \delta_{m, n}
$$

where $i=\sqrt{-1}$ and $\delta_{m, n}$ denotes the Kronecker delta.

The explicit form of the orthogonal polynomial is given by Carlitz [4] as

$$
\begin{aligned}
\lambda_{n}^{(k)}(x) & =\sum_{r=0}^{n} 2^{r}\left(\begin{array}{c}
\frac{1}{2}(x-k) \\
r
\end{array}\right)\left(\begin{array}{c}
n+k-1 \\
n-r
\end{array}\right) \\
& =(-1)^{n}(k)_{n} 2_{1}^{F}\left[-n, \frac{1}{2}(x+k) ; k ; 2\right] .
\end{aligned}
$$

The last result follows easily from the following well-known generating function for the Gaussian hypergeometric function ${ }_{2} F_{1}[7, p .82]$ 


$$
\begin{aligned}
(1+z)^{b-c}[1+(1-x) z]^{-b}= & \left.\sum_{n=0}^{\infty} \int_{n}^{-c}\right){ }_{2} F_{1}[-n, b ; c ; x] z^{n} \\
& |z|<1,|z-z x|<1 .
\end{aligned}
$$

A related system of polynomials has been discussed by Bateman [1] who referred to them as the Mittag-Leffler polynomials. It happens that both the polynomials discussed by Bateman and Carlitz are but special cases of the system of orthogonal polynomials first discussed by Meixner [11] and later independently by Pollaczek [12]. Following the notation of [8, p. 219] (See also [5, p. 184]), the Meixner-Pollaczek polynomials are given explicitly by

$$
\mathrm{P}_{\mathrm{n}}^{(\alpha)}(\mathrm{x} ; \phi)=\frac{(2 \alpha) \mathrm{n}}{\mathrm{n} !} \mathrm{e}^{\mathrm{in} \phi}{ }_{2} \mathrm{~F}_{1}\left[-\mathrm{n}, \alpha+i x ; 2 \alpha ; 1-\mathrm{e}^{-2 i \phi}\right]
$$

where $\alpha>0,0<\phi<\pi$ and $-\infty<x<\infty$.

These polynomials are orthogonal with respect to the weight function

$$
\omega^{(\alpha)}(x ; \phi)=\frac{(2 \sin \phi)^{2 \alpha-1}}{\pi} \mathrm{e}^{-(\pi-2 \phi) x}|\Gamma(\alpha+i x)|^{2} \text {. }
$$

The orthogonality relation is given by

$$
\int_{-\infty}^{\infty} \omega^{(\alpha)}(x ; \phi) \mathrm{P}_{\mathrm{m}}^{(\alpha)}(\mathrm{x} ; \phi) \mathrm{P}_{\mathrm{n}}^{(\alpha)}(\mathrm{x} ; \phi) \mathrm{dx}=\frac{\Gamma(2 \alpha+\mathrm{n})}{\mathrm{n} !} \operatorname{cosec} \phi \delta_{\mathrm{m}, \mathrm{n}} \text {. }
$$

A generating function for $\mathrm{P}_{\mathrm{n}}^{(\alpha)}(\mathrm{x} ; \phi)$ is

$$
\sum_{n=0}^{\infty} t^{n_{P}(\alpha)}(x ; \phi)=\left(1-t e^{i \phi}\right)^{-\alpha+i x}\left(1-t e^{-i \phi}\right)^{-\alpha-i x},|t|<1 .
$$

It is clear when comparing (7) with (8) or (5) with (9) that

$$
(-i){ }_{n}^{n}(k)(i x)=n ! P_{n}^{(k / 2)}(x / 2 ; \pi / 2), k=1,2, \ldots ; n=0,1,2, \ldots
$$

and thus $\lambda_{n}^{(k)}(x)$ may be regarded as a special case of the Meixner-Pollaczek polynomials . 


\section{A BILINEAR SUMMATION FORMULA}

From the generating function in (5) it is immediately clear that $\lambda_{n}^{(k)}(x)$ satisfies the following so-called Runge-type identity

$$
\begin{array}{r}
\lambda_{n}^{\left(k_{1}+k_{2}\right)}\left(x_{1}+x_{2}\right)=\sum_{r=0}^{n} \int_{r}^{n} \lambda_{r}^{\left(k_{1}\right)}\left(x_{1}\right) \lambda_{n-r}^{\left(k_{2}\right)}\left(x_{2}\right), k_{1}, k_{2}=1,2,3, \ldots \\
\text { and all } n .
\end{array}
$$

It has been shown that the result in (10) is both necessary and sufficient for the joint p.d.f. in (2) to possess a bilinear expansion (also called a canonical expansion in statistical 1iterature) of the form [6]

$$
\mathrm{p}\left(\mathrm{x}_{1}, \mathrm{x}_{2}\right)=\mathrm{g}\left(\mathrm{x}_{1}\right) \mathrm{h}\left(\mathrm{x}_{2}\right) \sum_{\mathrm{r}=0}^{\infty} \rho_{\mathrm{n}} \theta_{\mathrm{n}}\left(\mathrm{x}_{1}\right) \phi_{\mathrm{n}}\left(\mathrm{x}_{2}\right)
$$

where the canonical variables $\left\{\theta_{n}(x)\right\} \quad\left(\left\{\phi_{n}(x)\right\}\right)$ are a complete set of orthonormal polynomials with weight function $g(x)(h(x))$. The canonical correlation is

$$
\rho_{n}=E\left[\theta_{n}\left(x_{1}\right) \phi_{n}\left(x_{2}\right)\right]
$$

where $\mathrm{E}$ denotes the expectation operation.

For the joint p.d.f. in (2) with the equal marginal p.d.f.'s given in (3), we note that the canonical variable in this case is

$$
\theta_{n}(x)=\phi_{n}(x)=\frac{i^{-n}}{\sqrt{n !(2)_{n}}} \lambda_{n}^{(2)}(i x) .
$$

The canonical correlation is

$$
\begin{aligned}
\rho_{n} & =E\left[\theta_{n}\left(x_{1}\right) \phi_{n}\left(x_{2}\right)\right] \\
& =\frac{(-1)^{-n}}{n !(2)_{n}} E\left\{\lambda_{n}^{(2)}\left[i\left(v_{1}+v_{2}\right)\right] \lambda_{n}^{(2)}\left[i\left(v_{2}+v_{3}\right)\right]\right\} \\
& \left.\left.=\frac{(-1)^{-n}}{n !(2)_{n}} \sum_{s=0}^{n} \sum_{r=0}^{n} \int_{s}^{n}\right) \int_{r}^{n}\right\} E\left[\lambda_{r}^{(1)}\left(i v_{1}\right)\right] .
\end{aligned}
$$




$$
\begin{aligned}
& \quad . E\left[\lambda_{n-r}^{(1)}\left(i v_{2}\right) \lambda_{s}^{(1)}\left(i v_{2}\right)\right] E\left[\lambda_{n-s}^{(1)}\left(i v_{3}\right)\right] \\
& =\frac{(-1)^{-n}}{n !(2)_{n}} E\left[\lambda_{n}^{\left.(1) \cdot\left(i v_{2}\right)\right]^{2}}\right. \\
& =\frac{1}{n+1} .
\end{aligned}
$$

We therefore have the following interesting bilinear sumation formula for the Meixner-Pollaczek polynomials

$$
\begin{aligned}
\sum_{n=0}^{\infty} \frac{(-1)^{n}}{[(n+1) !]^{2}} \lambda_{n}^{(2)}\left(i x_{1}\right) \lambda_{n}^{(2)}\left(i x_{2}\right)= & \sinh \frac{\pi x_{1}}{2} \sinh \frac{\pi x_{2}}{2} \operatorname{sech} \frac{\pi\left(x_{2}-x_{1}\right)}{2} /\left(2 x_{1} x_{2}\right) \\
& -\infty<x_{1}<\infty \text { and }-\infty<x_{2}<\infty .
\end{aligned}
$$

\section{A GENERALIZATION}

Consider the following more general scheme of additive random variables as in [9].

Let $\left\{\xi_{i}\right\}$ for $i=1,2, \ldots, n-m,\left\{n_{i}\right\}$ for $i=1,2, \ldots, m$

and $\left\{\zeta_{i}\right\}$ for $i=1,2, \ldots, n_{2}-m$ where $1 \leqslant m<\min \left(n_{1}, n_{2}\right)$

be $\left(n_{1}+n_{2}-m\right)$ mutually independent random variables each having the p.d.f. given in (1).

Define

$$
\begin{aligned}
\mathrm{U}=\sum_{\mathrm{i}=1}^{\mathrm{n}_{1}-\mathrm{m}} \xi_{\mathrm{i}}, \quad \mathrm{v} & =\sum_{\mathrm{i}=1}^{\mathrm{m}} \eta_{\mathrm{i}}, \quad \mathrm{w}=\sum_{\mathrm{i}=1}^{\mathrm{n}_{2}-\mathrm{m}} \zeta_{\mathrm{i}} \\
\mathrm{x}_{1} & =\mathrm{U}+\mathrm{v} \\
\mathrm{x}_{2} & =\mathrm{v}+\mathrm{w} .
\end{aligned}
$$

It is clear that the joint characteristic function $\phi\left(\omega_{1}, \omega_{2}\right)$ of $x_{1}$ and $\mathrm{x}_{2}$ is

$$
\phi\left(\omega_{1}, \omega_{2}\right)=E\left[\exp \left(i \omega_{1} x_{1}+i \omega_{2} x_{2}\right)\right]
$$




$$
\begin{aligned}
& =E\left\{\exp \left[i \omega_{1} U+i \omega_{2} W+i\left(\omega_{1}+\omega_{2}\right) v\right]\right\} \\
& =\operatorname{sech}^{n_{1}-m}\left(\frac{\omega_{1}}{2}\right) \operatorname{sech}^{n_{2}-m}\left(\frac{\omega_{2}}{2}\right) \operatorname{sech}^{m}\left(\frac{\omega_{1}+\omega_{2}}{2}\right) \\
& E\left[e^{i \omega \xi_{i}}\right]=E\left[e^{i \omega n_{j}}\right]=E\left[e^{i \omega \xi_{k}}\right] \\
& =\int_{-\infty}^{\infty} e^{i \omega v} \operatorname{sech} \pi v d v \\
& =\operatorname{sech(\frac {\omega }{2})} \text { for } 1 \leqslant i \leqslant n_{1}-m, \\
& 1 \leqslant j \leqslant m, \\
& 1 \leqslant k \leqslant n_{2}-m .
\end{aligned}
$$

The joint p.d.f. in question is therefore

$$
\begin{array}{r}
p\left(x_{1}, x_{2}\right)=\frac{1}{4 \pi^{2}} \int_{-\infty}^{\infty} \int_{-\infty}^{\infty} e^{-i \omega_{1} x_{1}-i \omega_{2} x_{2}} \operatorname{sech}^{n_{1}-m^{-}\left(\frac{\omega_{1}}{2}\right) \operatorname{sech}^{n_{2}-m}\left(\frac{\omega_{2}}{2}\right) .} \\
\operatorname{sech}^{m}\left(\frac{\omega_{1}+\omega_{2}}{2}\right) d \omega_{1} d \omega_{2}
\end{array}
$$

and the marginal p.d.f.'s for $x_{1}$ and $x_{2}$ are respectively

$$
\begin{aligned}
g\left(x_{1}\right) & =\frac{1}{2 \pi} \int_{-\infty}^{\infty} e^{-i \omega_{1} x_{1}} \operatorname{sech}^{n_{1}}\left(\frac{\omega_{1}}{2}\right) d \omega_{1} \\
& =\frac{1}{\pi} \frac{2^{n_{1}-1}}{\left(n_{1}-1\right) !}\left|\Gamma\left(\frac{n_{1}}{2}+i x_{1}\right)\right|^{2} \\
h\left(x_{2}\right) & =\frac{1}{2 \pi} \int_{-\infty}^{\infty} e^{-i \omega_{2} x_{2}} \operatorname{sech}^{n_{2}}\left(\frac{\omega_{2}}{2}\right) d \omega_{2} \\
& =\frac{1}{\pi} \frac{2^{n_{2}-1}}{\left(n_{2}-1\right) !}\left|\Gamma\left(\frac{n_{2}}{2}+i x_{2}\right)\right|^{2}
\end{aligned}
$$

on using the fact that $[3, p .31]$ 


$$
\int_{-\infty}^{\infty} e^{-i v x}[\operatorname{sech}(\beta x+\gamma)]^{\mu+1} d x=\frac{2^{\mu}}{\beta} \frac{\left|\Gamma\left(\frac{1+\mu+i v / \beta}{2}\right)\right|^{2}}{\Gamma(\mu+1)} e^{i v \gamma / \beta} .
$$

The respective canonical variables are

$$
\begin{aligned}
& \theta_{n}\left(x_{1}\right)=\frac{i^{-n}}{\sqrt{n !\left(n_{1}\right)_{n}}} \lambda_{n}^{\left(n_{1}\right)}\left(i x_{1}\right) \\
& \phi_{n}\left(x_{2}\right)=\frac{i^{-n}}{\sqrt{n !\left(n_{2}\right)_{n}}} \lambda_{n}^{\left(n_{2}\right)}\left(i x_{2}\right) .
\end{aligned}
$$

By a repeated application of the Runge-type identity in (10) analogous to the derivation leading to the result in (11), it may be shown that the canonical correlation in this case is

$$
\rho_{n}=\frac{(m)}{\sqrt{\left(n_{1}\right)_{n}\left(n_{2}\right)_{n}}}, \quad 1 \leqslant m<\min \left(n_{1}, n_{2}\right) \text {. }
$$

On the other hand, note that from (14)

$$
\begin{aligned}
& \frac{1}{4 \pi^{2}} \int_{-\infty}^{\infty} \int_{-\infty}^{\infty} e^{i \omega_{1} x_{1}-i \omega_{2} x_{2}} \operatorname{sech}^{m}\left(\frac{\omega_{1}+\omega_{2}}{2}\right) d \omega_{1} d \omega_{2} \\
& =\frac{2^{m-1}}{\pi(m-1) !}\left|\Gamma\left(\frac{m}{2}+i x_{1}\right)\right|^{2} \cdot \frac{1}{2 \pi} \int_{-\infty}^{\infty} \exp \left[-i \omega_{2}\left(x_{2}-x_{1}\right)\right] d \omega_{2} \\
& =\frac{2^{m-1}}{\pi(m-1) !}\left|\Gamma\left(\frac{m}{2}+i x_{1}\right)\right|^{2} \delta\left(x_{2}-x_{1}\right)
\end{aligned}
$$

where $\delta(x)$ denotes the Dirac delta function.

A double convolution operation applied to (13) then yields the following expression for $p\left(x_{1}, x_{2}\right)$

$$
\begin{aligned}
p\left(x_{1}, x_{2}\right)= & \frac{2^{n_{1}+n_{2}+m-3}}{\pi^{3}(m-1) !\left(n_{1}-m-1\right) !\left(n_{2}-m-1\right) !} \int_{-\infty}^{\infty} \int_{-\infty}^{\infty}\left|\Gamma\left(\frac{n_{1}-m}{2}+i u\right)\right|^{2} . \\
& \left|\Gamma\left(\frac{n_{2}-m}{2}+i v\right)\right|^{2}\left|\Gamma\left(\frac{m}{2}+i\left(x_{1}-u\right)\right)\right| \delta\left(x_{2}-v-x_{1}+u\right) d u d v
\end{aligned}
$$




$$
\begin{aligned}
& =\frac{2^{n_{1}+n_{2}+m-3}}{\pi^{3}(m-1) !\left(n_{1}-m-1\right) !\left(n_{2}-m-1\right) !} \int_{-\infty}^{\infty}\left|\Gamma\left(\frac{n_{1}-m}{2}+i u\right)\right|^{2} . \\
& \left|\Gamma\left(\frac{m}{2}+i\left(x_{1}-u\right)\right)\right|^{2}\left|\Gamma\left(\frac{n_{2}-m}{2}+i\left(x_{2}-x_{1}+u\right)\right)\right|^{2} d u .
\end{aligned}
$$

Finally, the result in (15) may be rewritten into the following Barnes type contour integral

$$
\begin{aligned}
p\left(x_{1}, x_{2}\right)= & \frac{2^{n_{1}+n_{2}+m-2}}{\pi^{2}(m-1) !\left(n_{1}-m-1\right) !\left(n_{2}-m-1\right) !} \cdot \frac{1}{2 \pi i} \int_{-i \infty}^{-i \infty} \Gamma\left(\frac{n_{1}-m}{2}+s\right) \Gamma\left(\frac{m}{2}-i x_{1}+s\right) . \\
& \Gamma\left(\frac{n_{2}-m}{2}+i\left(x_{2}-x_{1}\right)+s\right) \Gamma\left(\frac{n_{1}-m}{2}-s\right) \Gamma\left(\frac{m}{2}+i x_{1}-s\right) \Gamma\left(\frac{n_{2}-m}{2}-i\left(x_{2}-x_{1}\right)-s\right) d s
\end{aligned}
$$

which may be evaluated in terms of a sum of ${ }_{3} F_{2}$ functions [13, p. 133] or, perhaps more conveniently, in terms of Meijer's G-function as follows [7, Sec. 5.3]

$$
\begin{aligned}
\mathrm{p}\left(\mathrm{x}_{1}, \mathrm{x}_{2}\right)= & \frac{2^{\mathrm{n}_{1}+\mathrm{n}_{2}-\mathrm{m}-2}}{\pi^{2}(\mathrm{~m}-1) !\left(\mathrm{n}_{1}-\mathrm{m}-1\right) !\left(\mathrm{n}_{2}-\mathrm{m}-1\right) !} . \\
& \mathrm{G}_{3,3,3}^{3,3}\left(\begin{array}{ccc}
1-\frac{\mathrm{n}_{1}-\mathrm{m}}{2}, & 1-\frac{\mathrm{m}+i \mathrm{x}_{1},}{2} & 1-\frac{\mathrm{n}_{2}-\mathrm{m}}{2}-i\left(\mathrm{x}_{2}-\mathrm{x}_{1}\right) \\
\frac{\mathrm{n}_{1}-\mathrm{m}}{2}, & \frac{m}{2}+i \mathrm{x}_{1}, & \frac{\mathrm{n}_{2}-\mathrm{m}}{2}-i\left(\mathrm{x}_{2}-\mathrm{x}_{1}\right)
\end{array}\right) .
\end{aligned}
$$

The existence of a diagonal expansion then implies the following summation formula

$$
\begin{aligned}
& \sum_{n=0}^{\infty} \frac{(-1)^{n}(m) n}{\left(n_{1}\right)_{n}\left(n_{2}\right) n !} \lambda_{n}^{\left(n_{1}\right)}\left(x_{1}\right) \lambda_{n}^{\left(n_{2}\right)}\left(x_{2}\right) \\
& =\frac{\left(n_{1}-1\right) !\left(n_{2}-1\right) !}{2^{m}(m-1) !\left(n_{1}-m-1\right) !\left(n_{2}-m-1\right) !} \cdot \frac{1}{\Gamma\left(\frac{n_{1}}{2}+x_{1}\right) \Gamma\left(\frac{n_{1}}{2}-x_{1}\right) \Gamma\left(\frac{n_{2}}{2}+x_{2}\right) \Gamma\left(\frac{n_{2}}{2}-x_{2}\right)} .
\end{aligned}
$$




$$
\text { - } G_{3,3}^{3,3}\left(\begin{array}{ccc}
1-\frac{n_{1}-m}{2}, & 1-\frac{m}{2}+x_{1}, & 1-\frac{n_{2}-m}{2}-\left(x_{2}-x_{1}\right) \\
\frac{n_{1}-m}{2}, & \frac{m}{2}+x_{1}, & \frac{n_{2}-m}{2}-\left(x_{2}-x_{1}\right)
\end{array}\right)
$$

for $1 \leqslant m<\min \left(n_{1}, n_{2}\right),-\infty<x_{1}<\infty,-\infty<x_{2}<\infty$.

It is perhaps interesting to note in passing that a comparison of the two results in (12) and (16) allows us to deduce the following special case of the G-function, viz.

$$
G_{3,3}^{3,3}\left(\begin{array}{lll}
\frac{1}{2}, & \frac{1}{2}+x_{1}, & \frac{1}{2}+x_{1}-x_{2} \\
\frac{1}{2}, & \frac{1}{2}+x_{1}, & \frac{1}{2}+x_{1}-x_{2}
\end{array}\right)=\frac{\pi^{2}}{4} \sec \frac{\pi x_{1}}{2} \sec \frac{\pi x_{2}}{2} \sec \frac{\pi\left(x_{2}-x_{1}\right)}{2} .
$$

ACKNOWLEDGEMENT. The author is grateful to the referee for his helpful comments, in particular regarding the connection of the system of orthogonal polynomials discussed by Carlitz with that of the Meixner-Pollaczek polynomials.

\section{REFERENCES}

1. Bateman, H. The Polynomials of Mittag-Leffler, Proc. Nat. Acad. Sci. (U.S.A.) 26 (1940) 491-496.

2. Baten, W.D. The Probability Law for the Sum of $n$ Independent Variables, each subject to the Law $(1 /(2 \mathrm{~h})) \operatorname{sech}(\pi \mathrm{x} /(2 \mathrm{~h}))$, Bull. Amer. Math. Soc. 40 (1934) 284-290.

3. Buchholz, H. The Confluente Hypergeometric Functions, Springer Verlag, New York, 1969.

4. Carlitz, L. Bernoulli and Euler Numbers and Orthogonal Polynomials, Duke Math. J. 26 (1959) 1-15.

5. Chihara, T.S. An Introduction to Orthogonal Polynomials, Gordon and Breach, New York, 1978.

6. Eagleson, G.K. and H.O. Lancaster. The Regression System of Sums with Random Elements in Common, Aust. J. Statist. 9 (1967) 119-125.

7. Erdélyi, A., A. Magnus, F. Oberhettinger and F. Tricomi. Higher Transcendental Functions, Vo1. 1, McGraw-Hi11, New York, 1953.

8. Erdélyi, A., A. Magnus, F. Oberhettinger and F. Tricomi. Higher Transcendental Functions, Vo1. 2, McGraw-Hil1, New York, 1953. 
9. Hamden, M.A. Canonical Expansion of the Bivariate Binomial Distribution with Unequal Marginal Indices, Int. Statist. Rev. 40 (1972) 277-280.

10. Harkness, W.L. and M.L. Harkness. Generalized Hyperbolic Secant Distributions, J. Amer. Statist. Assoc. 63 (1968) 329-337.

11. Meixner, J. Orthogonale Polynomsysteme mit einem besonderen Gestalt der erzeugenden Funktion, J. London Math. Soc. 9 (1934) 6-13.

12. Pollaczek, F. Sur une famille de polynômes orthogonaux qui contient les polynômes d'Hermite et de Laguerre comme cas limites, C.R. Acad. Sci., Paris 230 (1950) 1563-1565.

13. Slater, L.J. Generalized Hypergeometric Functions, Cambridge University Press, London, 1966. 


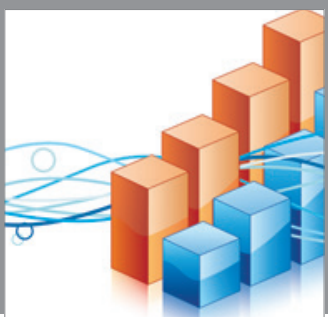

Advances in

Operations Research

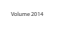

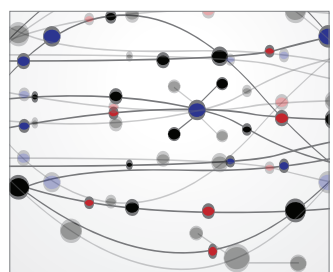

\section{The Scientific} World Journal
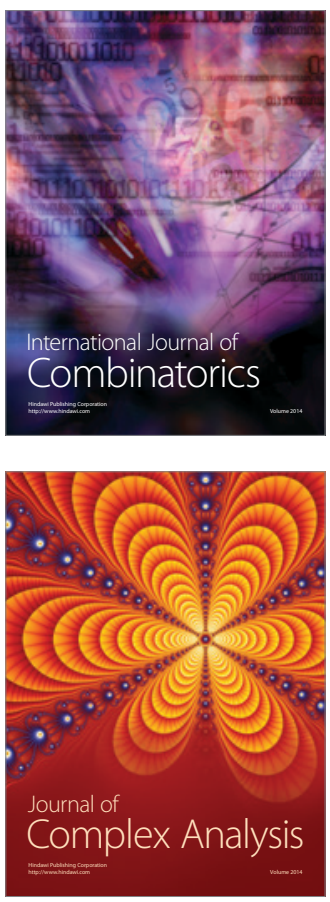

International Journal of

Mathematics and

Mathematical

Sciences
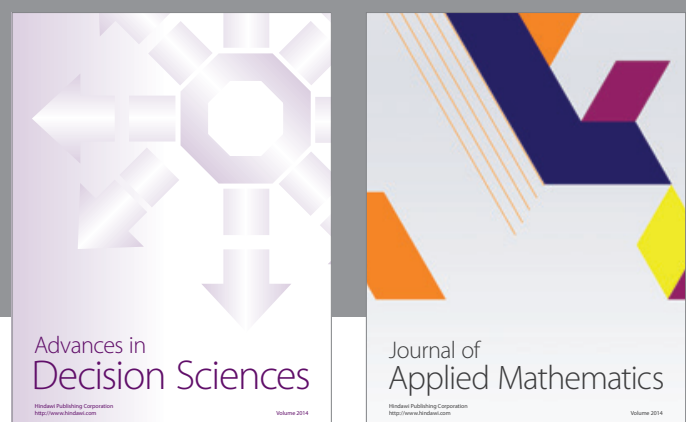

Journal of

Applied Mathematics
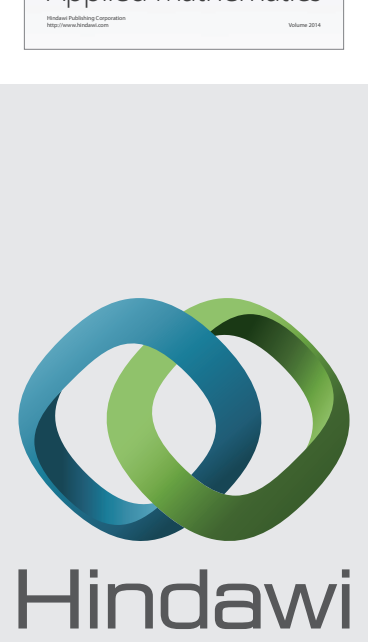

Submit your manuscripts at http://www.hindawi.com
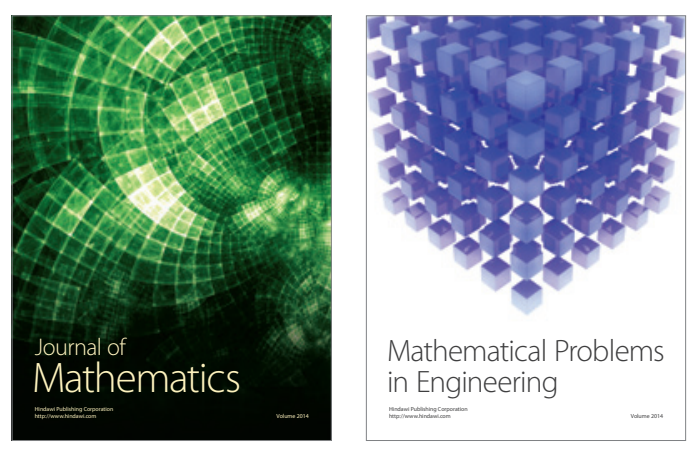

Mathematical Problems in Engineering
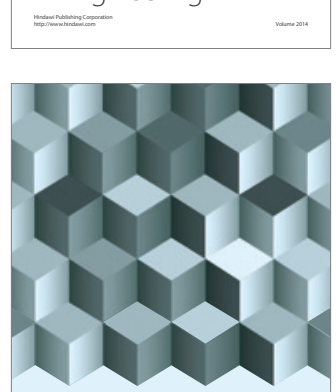

Journal of

Function Spaces
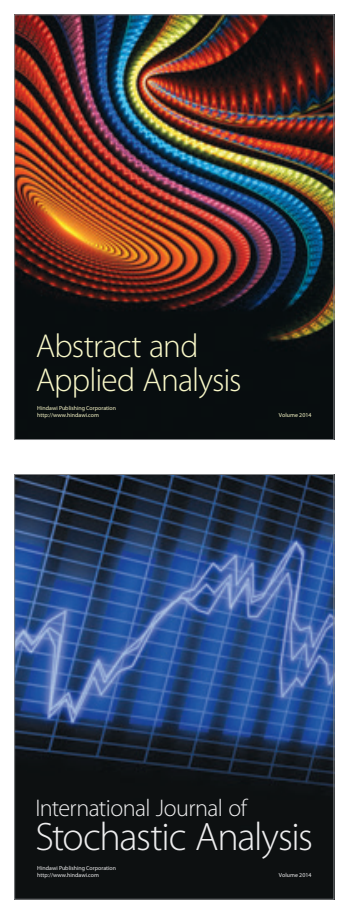

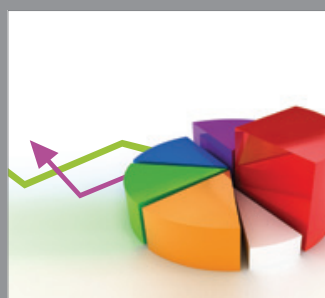

ournal of

Probability and Statistics

Promensencen
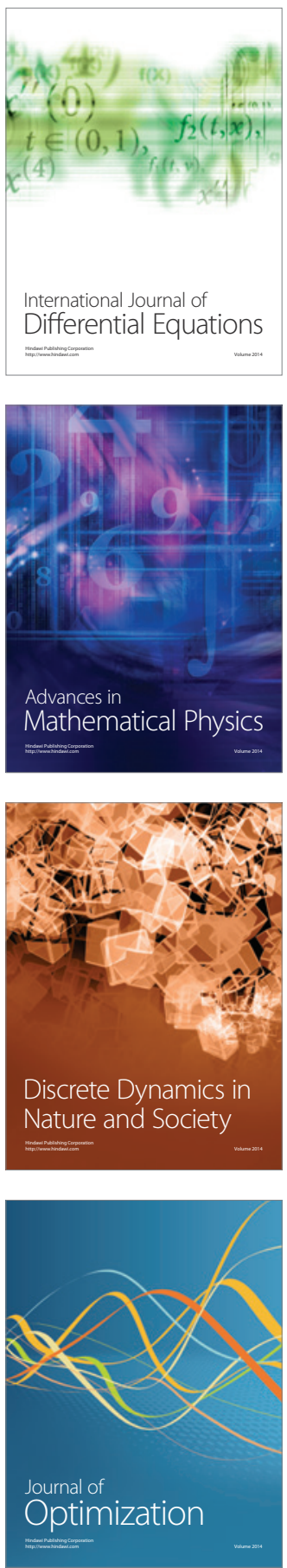